\title{
Las asignaturas de Relaciones Públicas en los Grados catalanes del E.E.E.S. al cierre de su primer ciclo (2009-13)
}

\author{
Kathy Matilla \\ Universidad Ramón Llull-URL \\ kathyms@blanquerna.url.edu \\ Salvador HERNÁNDEZ \\ Universidad Católica San Antonio-UCAM \\ shernandez@ucam.edu
}

\section{Resumen}

Las asignaturas de Relaciones Públicas (R.R.P.P.) en los estudios universitarios de Grado en Cataluña, al finalizar su primer ciclo completo, no han sido objeto de cambios sustanciales en relación a las Licenciaturas anteriores al E.E.E.S.

Palabras clave: Asignaturas; Cataluña; Espacio Europeo de Educación Superior (E.E.E.S.); Grados; Relaciones Públicas; Universidad. Guerra Mundial; mujer; estereotipos; historia de las mujeres.

The Public Relations courses in Catalonian Degrees adapted to the European Higher Education Area-EHEA at the end of its first cycle (2009-13)

\begin{abstract}
The courses of Public Relations (PR) in the Catalonian university degrees have not changed substantially, at the end of its first full cycle, in relation to the degrees before the implementation of the European Higher Education Area (EHEA).
\end{abstract}

Key words: Courses, Catalonia, European Higher Education Area (EHEA), Public Relations, University.

\section{Referencia normalizada:}

Matilla, K. y Hernández, S. (2013) Las asignaturas de Relaciones Públicas en los Grados catalanes del E.E.E.S. al cierre de su primer ciclo (2009-13). Historia y Comunicación Social. Vol. 18 № Especial Octubre. Págs. 279-293

Sumario: 1. Introducción; 2. Marco Teórico; 3. Metodología; 4. Resultados y Discusión; 5. Conclusiones; Referencias Bibliográficas.

\section{Introducción}

A partir de una descripción de la oferta de las asignaturas de R.R.P.P. en los Grados de Publicidad y R.R.P.P. del sistema universitario catalán adaptado al E.E.E.S. durante el periodo 2009-13, es decir, desde su inauguración hasta la finalización de su primer ciclo completo, hemos contextualizado nuestro objeto de estudio con un 
marco teórico que recoge los resultados de los principales estudios elaborados por investigadores y profesionales especializados en R.R.P.P., que describen -o de los que se infiere- el perfil profesional idóneo para desarrollar la praxis profesional, para compararlos con las competencias específicas que, sobre R.R.P.P., pueden llegar a adquirir los egresados interesados en especializarse en dicha disciplina una vez cursados los programas formativos de Grado ofertados por el sistema universitario catalán. A continuación presentamos los resultados del balance general del periodo analizado.

\section{Marco teórico}

Los estudios identificados y analizados son obra de profesionales y académicos especializados en R.R.P.P., tanto de ámbito nacional como internacional, y nos permitirán valorar si las competencias a adquirir por los futuros graduados en dicha disciplina, una vez cursados los programas formativos ofertados por las universidades catalanas, son los más apropiados para el logro de un adecuado desempeño en su futura inserción profesional.

Estos son los 47 estudios que hemos considerado y estudiado:

- Profesionales internacionales: 1) VII World Public Relations Forum de la Global Alliance for Public Relations and Communication Management (2012); 2) European Communication Monitor (2007, 2008, 2009, 2010, 2011, 2012); ICCC-International Corporate Communication Compass for Europe de la EUPRERA; 3) The Melbourne Mandate de la Global Alliance for PR and Communication Management (2012); 4) Stockholm Accords de la Global Alliance for PR and Communication Management (2010); 5) diversos documentos del Corporate Excellence-Centre for Reputation Leadership (2012a, 2012b, 2012c, 2012d, 2012e, 2013a y 2013b).

- Profesionales españoles: 6) El estado de la comunicación en España de Dircom (2010); 7) El Barómetro CyD: el papel de la Universidad en la empresa de la Fundación CYD-Conocimiento y Desarrollo (2008); 8) La Comunicación y las R.R.P.P.: Radiografía del sector de ADECEC (2005 y 2008).

- Académicos internacionales: 9) Resources for PR Educators for Teaching Research, Measurement and Evaluation de la IPR Education in PR Comission (2012); 10) PR in a New Age de Botan y Hazleton (2006); 11) Overview of PR in South America de Ferrari (2009); 12) PR Education de L'Etang y Pieckza (2006); 13) PR as relationship management. A relational approach to the study and practice of PR de Ledingham y Bruning (2000); 14) PR Knowledge and Professionalism: Challenges to Educators and Practitioners de Wright y Turk (2007); 15) PR State of the Field de Botan y Taylor (2004); 16) Advancement for PR Role Models de Broom y Dozier (1986); 17) Evolution of the Manager Role in PR Practice de Dozier y Broom (1995); 18) A generic model for vocationally oriented PR education in globalised contexts de Ferreira y 
Verwey (2004); 19) Today's PR: an Introduction de Heath y Coombs (2006); 20) PR in Portugal. An Analysis of the Profession through the Undergraduate Curriculum de Gonçalves (2009); 21) PR Education in Britain: An Historical Review in the Context of Professionalism de L'Etang (1999); 22) Toward a Global Curriculum: A Summary of Concerning PR Education, Professionalism and Globalization de la Leeds Metropolitan University y la Global Alliance (2008); 23) International Paradigms: The Latin American School of PR de Molleda (2000); 24) The Dire Need for Multiculturalism in PR Education: An Asian Perspective de Sriramesh (2002); 25) PR and the New Golden Age of Spain: a Confluence of Democracy, Economic Development and the Media de Tilson y Pérez (2003); 26) PR Higher Education. A Russian experience de Azarova (2003); 27) The Professional Bond: PR Education for the 21st Century de la Commission on PR Education (2006); 28) Profiling the PR Undergraduate / Bachelor and Graduate / Master Programmes in Europe and Beyond de Cotton y Tench (2009); 29) The History and Development of PR Education in Turkey: A Quick Glimpse de Gorpe (2009); 30) Resources for PR Educators for Teaching Research, Measurement and Evaluation del IPR y O'Neil (2012); 31) Approaches to and Instruments of PR: Higher Education in the Czech Republic de Pirozek y Heskova (2003); 32) PR, Marketing and Communications in Eastern European Universities de Spacal (2007); 33) A First Look: an In-Depth Analysis og Global PR Education. PR Curriculum and Instructor for 20 Countries de Tooth y Aldoory para la PRSA Foundation, la Global Alliance for PR and Communications Management y la Commission on PR Education; 34) Understanding Chinese PR Education: A Critical and Cultural Perspective de Zhang (2009); 35) PR Education. An Instrument for the Transformation and Development of Human Resources de Zlateva (2003).

- Académicos nacionales: 36) Lo que hay que tener de Lalueza (2009); 37) Center and Periphery: Two Speeds for the Implementation of PR in Spain de Huertas y Fernández Cavia (2006); 38) El Espacio Europeo de Educación Superior: la razón instrumental de una universidad al servicio de la empresa de Pardo y Gracía Toribio (2008); 39) Undergraduate PR Education in Spain: Endangered Species? de Xifra (2007); 40) Definición empírico-operativa del "practicum» de los estudiantes universitarios de R.R.P.P. y su vinculación con la realidad profesional en Cataluña, 1992-1997 de Magallón (1998).

- Contribuciones de las instituciones gubernamentales educativas comunitarias, españolas y catalanas: 41) About the Bologna Process del Benelux Bologna Secretariat (2010); 41) Ley Orgánica 4/2007, de 12 de abril, por la que se modifica la Ley Orgánica 6/2001, de 21 de diciembre, de Universidades, BOE $\mathrm{n}^{\mathrm{o}} 89$ de 13.04.07; 42) Propuestas para la renovación de las metodologías educativas de la universidad (2006) del Consejo de Coordinación Universitaria de la Secretaría de Estado de Universidades e Investigación del Ministerio de Educación; 43) Datos y cifras del Sistema Universitario. Curso 2009-10 (2010) del Ministerio de Educación; 44) Títulos de Grado en Comunicación: 
Libro Blanco (2005) de la ANECA; 45) Guía de Evaluación del diseño del Sistema de Garantía Interna de Calidad de la formación universitaria (2008) de la ANECA; 46) Directrius per al Desenvolupament del Marc per a la Verificació, el Seguiment, la Modificació i l'Acreditació de titulacions oficials (2010) de la AQU; y 47) Guia per a l'acreditació de les titulacions oficials de grau i màster (2013) de la AQU.

\section{Metodología}

Desarrollamos un rastreo sistemático de las páginas web de las universidades catalanas de la unidad de análisis, reconocidas por el Ministerio de Educación del estado español (estudio descriptivo transversal), con análisis de contenidos ${ }^{1}$ de las ofertas online de grados en los cursos académicos 2009-13, con el objetivo de realizar un análisis comparativo.

Se aplicó una plantilla de análisis con diversas variables: Universidad; Facultad; ciudad; tipología de Universidad (Pública / no Pública - Presencial / No presencial (online); denominación del Grado; denominación de las asignaturas (sólo de R.R.P.P.; sólo de Publicidad; mixtas de R.R.P.P. y Publicidad); clasificación de las asignaturas (obligatorias u optativas); curso; y módulo / mención en que se ofertaban dichas asignaturas.

El universo estaba configurado por las 12 universidades del sistema universitario catalán. La muestra de la unidad de análisis fueron las 10 universidades que ofertaban Grado de Publicidad y R.R.P.P. (o su equivalente). El estudio se ejecutó durante el primer semestre de cada curso académico, siempre entre el 10 de Septiembre y el 31 de Diciembre de cada año lectivo, a partir del buscador de la página web del Ministerio de Educación español

\section{Resultados y discusión}

La implantación de los Grados adaptados al E.E.E.S. es absoluta en la muestra analizada -con, mínimo, cuatro años de experiencia- y se observan escasas modificaciones a lo largo del periodo estudiado (2009-2013), aunque la más importante detectada en algunas universidades es la incorporación de las menciones, inspiradas en los itinerarios de especialización de las extintas Licenciaturas, que suelen agrupar las asignaturas optativas.

Los principales hallazgos por universidades al concluir la etapa de implantación masiva al E.E.E.S., fueron:

1 Ver metodología de análisis de contenidos en Krippendorff (1990) 
- U. Abat Oliba-CEU - Las asignaturas se presentan agrupadas por materias. En 3er. curso aparece la primera materia que plantea contenidos de R.R.P.P.

Asignaturas de R.R.P.P. de 3er. curso: Relaciones Públicas; Comunicación Institucional y Corporativa; Comunicación institucional y Corporativa; Medios de Comunicación; Sistemas de Comunicación de Marketing y Tecnologías de la Comunicación Publicitaria.

Asignaturas de R.R.P.P. $4^{\circ}$ curso: Comunicación y Propaganda Política; Organización de Eventos (se inscribe en Comunicación Publicitaria Avanzada).

El Grado tiene una duración de cinco años, en vez de cuatro, para añadirle la especialización -o mención- en International Business Program. Plantea 10 asignaturas, mayoritariamente de contenidos económicos y legales internacionales, y solo una oferta contenidos conjuntos de Publicidad y R.R.P.P., en inglés (Globalization: Markets \& Institutions) y, así, ninguna es específica de R.R.P.P.

Se observa desproporción entre los contenidos de R.R.P.P. y de Publicidad: 4 asignaturas de R.R.P.P. versus 15 de Publicidad, que superan cuantitativamente a las 12 de contenidos mixtos.

- U. Autónoma de Barcelona. Las asignaturas optativas vienen agrupadas en tres itinerarios de especialización - menciones-: Dirección Creativa, Dirección en Publicidad y R.R.P.P. y Dirección Estratégica en R.R.P.P. Las dos primeras únicamente ofertan una asignatura que comparte contenidos de Publicidad y R.R.P.P. (Técnicas de Negociación y Liderazgo), mientras que las demás asignaturas específicas de R.R.P.P. pertenecen al 3er. itinerario de R.R.P.P.

Se constata desproporción de contenidos: 20 asignaturas de Publicidad contra 17 de R.R.P.P. -que son prevalentemente optativas-. En las obligatorias y troncales la desproporción es significativamente superior: 11 de Publicidad y únicamente 2 de R.R.P.P.

- U. de Barcelona (centro E.S.R.P.-Escuela Superior de R.R.P.P., adscrito). Oferta 15 asignaturas específicas de R.R.P.P. contra 12 de Publicidad, siendo el único Grado de Cataluña que programa mayor número de asignaturas de R.R.P.P. que de Publicidad.

- U. de Gerona. Los contenidos del Grado se estructuran por módulos, subdivididos en materias y, éstas, en asignaturas. En los módulos no se especifican las asignaturas, sino las competencias de cada módulo, sus objetivos y unos contenidos-temario que, o bien se desglosan en 4 asignaturas independientes, o solo en 1 ó 2, lo que impide diferenciar las asignaturas de los módulos del plan de estudios, lo que supone una limitación a nuestra investigación.

En $4^{\circ}$ curso la UdG oferta 6 módulos: 1 específico de R.R.P.P. (Relaciones Institucionales) y 3 de contenidos mixtos (Producción Audiovisual y Gráfica 
aplicada a la Publicidad y las R.R.P.P.; Usos y efectos de las pantallas audiovisuales; y las Prácticas).

- U. Internacional de Cataluña. La mayoría de las asignaturas ofertadas son mixtas de Publicidad y R.R.P.P., con manifiesto desequilibrio entre las de Publicidad (11) y las de R.R.P.P. (3).

- U. Oberta de Cataluña. Agrupa las asignaturas optativas en itinerarios de especialización -menciones- y el Grado se diferencia al denominarse, genéricamente, de Comunicación. Las asignaturas de las 3 menciones de Publicidad y de R.R.P.P. son: Creatividad Publicitaria, Gestión Publicitaria y Comunicación Corporativa y R.R.P.P. Las otras 3 menciones aparecen vinculadas a las especializaciones de Periodismo y a la de Comunicación Audiovisual.

- U. Pompeu Fabra. En el número de asignaturas de Publicidad frente a las de R.R.P.P. se constata que las primeras (22) doblan a las segundas (11).

Las asignaturas optativas se agrupan en 4 itinerarios de especialización:

1. Estrategias de Comunicación Publicitaria.

2. Creatividad Publicitaria.

3. R.R.P.P.: Comunicación de Riesgo y de Crisis, Comunicación Interna, Estrategias de Acción Social, Protocolo de las organizaciones, Grupos de Presión y Grupos de Interés y Comunicación y Acontecimientos.

4. Análisis e Investigación en Publicidad y R.R.P.P.

- U. Ramón Llull. Se oferta una única asignatura de R.R.P.P. en el Grado, en 3er. Curso, y es una asignatura anual (Teoría y Técnicas de las R.R.P.P. I y II).

En $4^{\circ}$ curso no se programa ninguna asignatura específica de R.R.P.P., frente a las 13 de Publicidad y las 45 mixtas.

- U. Rovira i Virgili. Es, adicionalmente a la UB, una de las universidades que presenta mayor equilibrio cuantitativo entre asignaturas de R.R.P.P. (7) y de Publicidad (8).

- U. de Vic. Agrupa todas las asignaturas optativas en el último curso del Grado, en 4 menciones distintas:

\section{Business and Communication.}

2. Comunicación Global.

3. Publicidad.

4. R.R.P.P.: Dirección de Cuentas, Creación de Empresas de Comunicación, Lobbying Europeo e Internacional, Comunicación de Crisis y Conflicto, Patrocinio y Captación de Fondos, Responsabilidad Social Corporativa, Comunicación Interna, Expresión Oral y Comunicación No Verbal, Publi- 
cidad Institucional y Política, Ceremonial y Protocolo y Comunicación a las PYMES.

A pesar de que son prevalentes las asignaturas de Publicidad (23), las de R.R.P.P. (18) no muestran tanto desequilibrio como en otras universidades, siendo mayoritarias las asignaturas mixtas (38), como se constata habitual en la mayoría de los Grados analizados.

Al finalizar nuestro estudio, observamos que los contenidos específicos de R.R.P.P. -objeto de destacado interés en la literatura consultada-, que fundamentan la demanda de los futuros empleadores de los graduados en las facultades universitarias analizadas, no aparecen consignados, cualitativa ni cuantitativamente, en el número de asignaturas específicas de R.R.P.P. ofertadas por el sistema universitario catalán adaptado al E.E.E.S. en los programas formativos de los grados que constituyen nuestra unidad de análisis.

Por consiguiente, siguen de plena actualidad los postulados de Parés i Maicas (2006):

"Paradójicamente, a la relevancia que la Comunicación cobra en el campo profesional, su total legitimación científica y académica sigue sin encontrar acuerdos epistemológicos ni amplios consensos, haciendo de sus diversos trayectos teóricos e investigativos un constante y cíclico debate".

Parecen asimismo obviarse las recomendaciones publicadas en la literatura consultada en cuanto al perfil de conocimientos más adecuado para optar a la posición de Director de Comunicación en Cataluña, que aportan una valiosa información sobre las competencias y conocimientos demandados por el sector productivo y que proporcionaría a las universidades objeto de estudio una valiosa información para introducir posibles mejoras en sus ofertas curriculares.

\section{Conclusiones}

Es prevalente la inferioridad numérica de las asignaturas de R.R.P.P. de los Grados catalanes, frente a las de Publicidad, lo que conlleva, en la praxis profesional, la recepción de unos graduados con una insuficiente enseñanza por competencias en $\mathrm{R}$ R.R.P.P., disciplina en la que, los estudiantes que lo deseen, deberían poder especializarse en igualdad de condiciones que en Publicidad. Se origina, así: a) un incumplimiento de contrato de las facultades del sistema universitario catalán con sus "clientes" -los estudiantes que muestran vocación por las R.R.P.P.-, y b) una significativa falta de responsabilidad para con los empleadores que deben acogerlos e integrarlos tras su graduación. Académicamente, estos resultados no son consistentes con la literatura.

Podría inferirse, asimismo, un aparente incumplimiento de la Ley Orgánica 4/2007, de 12 de abril, por la que se modifica la Ley Orgánica 6/2001 de Universidades, de 21 de diciembre (BOE no 89 de 13.04.07, p. 16242), en relación al papel que deben jugar 
las agencias evaluadoras ANECA (España) y AQU (Cataluña), en lo que respecta a: a) el binomio autonomía-rendición de cuentas; b) la coordinación en los procesos de garantía de calidad y la definición de criterios de evaluación; c) la implicación de las universidades en la respuesta a las demandas de la sociedad y del sistema productivo; d) la mejor formación de sus graduados, para adaptarse a las demandas sociales y a las del sistema científico y tecnológico; e) que las universidades sean un motor para el avance del conocimiento y para el desarrollo social y económico del país; y f) los postulados del Libro Blanco de la ANECA, para el Grado en Publicidad y R.R.P.P. que, desde 2005, propugna que debe dotar de competencias, especialmente en lo que hace referencia a sus capacidades estratégicas, a los futuros

"profesionales que ejercen su actividad desde el ámbito de las organizaciones o desde una agencia o desde la empresa consultora, y profesionales responsables de la gestión estratégica de la Comunicación Corporativa" (ANECA, 2005: 333),

observándose, así, abundantes inconsistencias e incongruencias institucionales.

Paralelamente, todo parece apuntar a que, en materia de R.R.P.P., el sistema universitario catalán del E.E.E.S. se muestra reacio a la escucha y al diálogo para adaptarse a las exigencias de un colectivo profesional empleador en continuo proceso de mejora, obligado a asumir unos costes -tangibles e intangibles- que no les corresponden, que son la consecuencia de un débil aprendizaje universitario por competencias, producto de una oferta de estudios universitarios en buena medida obsoletos e infradotados y a incorporar a sus plantillas a nuevas promociones de graduados insuficientemente preparados.

Nuestras conclusiones son consistentes con Pérez García y Serrano Martínez (2012: 5):

"les empreses no reclamen ni tan sols mantenir la inversió pública en universitats. Al contrari, el discurs dominant reflecteix desconeixement i desconfiança vers la universitat" (Grau, 2012: 6); que "el potencial de las universidades y los universitarios no se aprovecha bien: hay problemas de eficacia y de eficiencia",

y congruentes con Piñuel Raigada et al. (2011: 5):

"hay división de opiniones sobre la necesidad de creación de un área [de conocimiento] propia. Entre los partidarios, la argumentación se centra en que la adscripción al campo de las Ciencias Sociales de distintas áreas de estudios de la Comunicación (v.g. del Periodismo, la Comunicación Audiovisual, la Publicidad, etc.) provoca diversos efectos perniciosos para el desarrollo de la investigación comunicacional".

Los resultados del balance final de esta primera etapa de adaptación al E.E.E.S. obligan a mantener vigentes las conclusiones anuales de los estudios realizados entre 2009 y $2012^{2}$, en aras a posibilitar: a) que el proceso de diseño y aprobación de las

2 "En las titulaciones universitarias catalanas regidas por el E.E.E.S. continua plenamente vigente que las asignaturas de R.P. se siguen encontrando, cuantitativamente hablando, relegadas a un segundo plano con respecto a las de Publicidad en los Grados de Publicidad y R.P. -o en sus equivalentes- y prácticamente igual a como estaban en las antiguas licenciaturas de las facultades de Comunicación 
titulaciones asegure una estructura curricular del programa formativo más satisfactoria; b) que el conjunto de módulos/materias del plan de estudios catalán sea coherente con las competencias de la titulación; c) que el contenido académico esté actualizado según la disciplina y el nivel de titulación; d) que las competencias sean consistentes con las de entidades nacionales e internacionales; e) que el diseño de la titulación (perfil de competencias y estructura del currículum) esté actualizado según los requisitos de la disciplina y responda al nivel formativo requerido en el M.E.C.E.S. ${ }^{3}$ (AQU, 2013: 18); f) que las universidades garanticen que sus actuaciones aseguren el logro de los objetivos de la formación que imparten, según el Real Decreto 1393/2007, modificado por el Real Decreto 861/2010; g) que los procedimientos de seguro externo de la calidad tengan en cuenta la eficacia de los procesos de seguro interno de la calidad, descritos en el apartado 1 de los Estándares y Directrices Europeos -ENQA, 2005- (AQU, 2013: 23); h) que los centros dispongan de un sistema de garantía interna de la calidad (S.G.I.C.) formalmente establecido e implantado, que asegure la calidad y la mejora continua de la titulación de forma eficiente; i) que el S.G.I.C. implantado en cada centro garantice la recogida de información y de resultados relevantes para la gestión eficiente de las titulaciones, en especial los resultados de aprendizaje y la satisfacción de los grupos de interés y que sea revisado periódicamente para analizar su adecuación y posible plan de mejora, para optimizarlo (AQU, 2013: 24); j) que las actividades formativas y el sistema de evaluación sean pertinentes, públicos y adecuados para certificar los aprendizajes reflejados en el nivel del M.E.C.E.S. del perfil de formación (AQU, 2013: 35) y en el Marco VSMA (AQU, 2010); y, finalmente, $\mathrm{k}$ ) que los empleadores muestren su satisfacción sobre la relación entre el perfil de formación esperado y el perfil de formación real (AQU, 2013: 40).

Nuestra investigación se ha desarrollado, curso a curso (Matilla y Hernández, 2010a, 2010b, 2011, 2012, 2013; Matilla y Xifra, 2009; Matilla, Losada y Hernández, 2010), por encargo de Dircom Cataluña, con el soporte institucional del Colegio de Publicitarios y Relaciones Públicas de Cataluña, las dos únicas asociaciones profesionales representativas de la práctica profesional de las R.R.P.P. en la comunidad autónoma catalana

\section{Referencias bibliograficas}

ADECEC (2005). La Comunicación y las Relaciones Públicas: Radiografía del sector. Barcelona: ADECEC.

catalanas anteriores al E.E.E.S., denunciadas por Xifra en 2007, y desde una perspectiva cualitativa, enfocadas a una visión fundamentalmente técnica (táctica) y escasa o nada estratégica" (Matilla, Losada y Hernández, 2009; Matilla y Hernández, 2010a; 2010b y 2011)”. (Matilla y Hernández, 2012: 264).

3 M.E.C.E.S. es el acrónimo del Marco Español de Calificaciones para la Educación Superior, que está alineado con el marco europeo construido sobre los Descriptores de Dublín. 
ADECEC (2008). La Comunicación y las Relaciones Públicas: Radiografía de un sector. Barcelona: ADECEC.

AE-IC-ASOCIACIÓN ESPAÑOLA DE INVESTIGACIÓN DE LA COMUNICACIÓN (2011). "Actas I Simposio Nacional de Grupos consolidados de Investigación en Comunicación". Abril. Madrid. http://es.scribd.com/doc/127756071/ InvestigarlaComu nicacionenEspana-Abril2011. [16.07.2013).

BOTAN, C. H.; HAZLETON, V. (2006). "Public Relations in a New Age". En BOTAN, C.H. y HAZLETON, V. (eds.), Public Relations Theory II. Mahwah (NJ): Erlbaum, pp. 1-18.

ANECA (2005). Títulos de Grado en Comunicación: Libro Blanco. http://www. aneca.es/. [03.05.2013].

(2008). Guía de Evaluación del diseño del Sistema de Garantía Interna de Calidad de la formación universitaria. http://www.aneca.es/media/166350/audit_ doc04_guiaevaluacion_080219.pdf. [06.05.2013].

AQU (2010). Directrius per al Desenvolupament del Marc per a la Verificació, el Seguiment, la Modificació i l'Acreditació del titulacions oficials. http://www.aqu. cat/doc/doc_13036660_1.pdf. [06.05.2013].

(2013). Guia per a l'acreditació de les titulacions oficials de grau i màster. http:// www.aqu.cat/doc/doc_38842795_1.pdf. [28.08.13].

AZAROVA, L. (2003). "PR Higher Education. A Russian Experience". En: Higher Education in Europe, 28, pp. 495-498. http:/www.ingentaconnect.com/content/ routledg/.../00000028/00000004. [19.06.2013].

BENELUX BOLOGNA SECRETARIAT (2010). About the Bologna Process. http:/ www.ond.vlaanderen.be/hogeronderwijs/bologna/about/. [09.06.2013].

COMMISSION ON PUBLIC RELATIONS EDUCATION (2006). The Professional Bond: Educación de R.P. para el s. XXI: el Vinculo Profesional. Informe de la Comisión de Educación en R.P. http://www.commpred.org/_uploads/report2-spanish.pdf. [16.07.2013].

BOTAN, G.M.; TAYLOR, M. (2004). "PR: State of the Field". En: Journal of Communication, December, pp. 645-661.

BROOM, G.M.; DOZIER, D.M. (1986). “Advancement for PR Role Models". En: Public Relations Review, 7 (1), pp. 37-56.

CORPORATE EXCELLENCE; VAN RIEL, C.B.M. (2013a). "What makes a Chief Communications Officer Excellent?" En: Insights-Documentos de Estrategia. http://www.corporateexcellence.org/index.php/Centro-de-conocimiento/ What-makes-a-Chief-Communications-Officer-Excellent. [08.08.2013].

CORPORATE EXCELLENCE (2013b). "Vincular estrategia con comunicación, objetivo del nuevo Dircom". En: Insights-Documentos de Estrategia. http://www. corporateexcellence.org/index.php/Centro-de-conocimiento/Vincular-estrategia-con-comunicacion-objetivo-del-nuevo-Dircom. [08.08.2013].

(2012a). "El papel del 'Chief Communications Officer' en la gestión de los intangibles". En: Insights-Documentos de Estrategia, 130, pp. 1-5. http://www.corporateexcellence.org/index.php/Centro-de-conocimiento/El-papel-del-Chief-Communications-Officer-en-la-gestion-de-los-intangibles. [08.08.2013]. 
(2012b). "Modelos académicos y de consultoría para evaluar el valor de las marcas". En: Insights-Documentos de Estrategia. http://ce.xsto.info/Centro-de-conocimiento/Modelos-academicos-y-de-consultoria-para-evaluar-el-valor-de-las-marcas. [08.08.2013].

(2012c). Jornada "Innovación en comunicación en el ámbito empresarial y los medios de comunicación". Universidad Complutense de Madrid. http:// corporateexcellence.org/index.php/Eventos/Jornada-Innovacion-en-comunicacion-en-el-ambito-empresarial-y-los-medios-de-comunicacion. [08.08.2013]. (2012d). "Dirigir la Comunicación en el marco de la nueva economía". En: Insights-Documentos de Estrategia. http://www.corporateexcellence.org/index.php/ Centro-de-conocimiento/Dirigir-la-comunicacion-en-el-marco-de-la-nueva-economia. [08.08.2013].

--- (2012e). "Reputación: el futuro de la Comunicación Corporativa". En: Insights-Documentos de Estrategia. http://www.corporateexcellence.org/index.php/ Centro-de-conocimiento/Reputacion-el-futuro-de-la-Comunicacion-Corporativa. [08.08.2013].

COTTON, A-M.; TENCH, R. (2009). Profiling the PR Undergraduate/Bachelor and Graduate/Master Programmes in Europe and Beyond. http://www.euprea.org/ webdate/downloads/112-berlinpresentationsurvey04april09.PDF. [11.08.2013].

DIRCOM (2010). El estado de la comunicación en España. http://www.slideshare. net/pacobarranco/estado-de-la-comunicacin-en-espaa-2010-estudio-de-dircom-4041488. [01.07.2013].

DOZIER, D.M.; BROOM, G.M. (1995). "Evolution of the Manager Role in PR Practice". En: Journal of Public Relations Research, 7 (1), pp. 3-26.

EUROPEAN COMMUNICATION MONITOR (2007, 2008, 2009, 2010, 2011, 2012). http://www. communicationmonitor.eu/. [15.06.2013].

FERRARI, M.A. (2009). "Overview of Public Relations in South America". En SRIRAMESH, K. y VERCIC, D. (eds.) (2009), The Global Public Relations Handbook: Theory, Research, and Practice. New York: Routledge, pp. 704-726.

FERREIRA, B.; VERWEY, S. (2004). "A generic model for vocationally oriented PR education in globalised contexts". En: Comunicare, 23, July, pp. 92-119.

FUNDACIÓN CYD-CONOCIMIENTO Y DESARROLLO (2008). "Barómetro CyD: el papel de la Universidad en la empresa". En: Colección Documentos CyD, $\mathrm{n}^{\circ}$ 9. www.fundacioncyd.org. [19.06.2013].

GLOBAL ALLIANCE FOR PR AND COMMUNICATION MANAGEMENT (2012). "The Melbourne Mandate", 7th World PR Forum. Melbourne (Australia). http://www.globalalliancepr.org/website/page/wprf-2012-melbourne. [16.08.2013].

--- (2010). Stockholm Accords. http://www.globalalliancepr.org/website/page/stockholm-accords. [16.08.2013].

GONÇALVES, G. (2009). "PR in Portugal. An Analysis of the Profession through the Undergraduate Curriculum”. En: Public Relations Review, 35, pp. 328-330. 
GORPE, S. (2009). "The History and Development of PR Education in Turkey: A Quick Glimpse”. UNESCO Conference, Sofía (Turquía). http:// serragorp@, yahoo.com. [20.07.2013].

GRAU VIDAL, F.X. (2012). La universidad pública española: Retos y prioridades en el marco de la crisis del primer decenio del s. XXI. Tarragona: Universidad Rovira i Virgili.

HEATH, R. L.; COOMBS, W. T. (2006). Today's PR: an Introduction. Thousand Oaks (CA): Sage.

HUERTAS, A.; FERnÁNDEZ CAVIA, J. (2006). "Center and Periphery: Two Speeds for the Implementation of PR in Spain". En: Public Relations Review, 32(2), pp. 110-117.

ICC-INTERNATIONAL CORPORATE COMMUNICATION COMPASS FOR EUROPE; EUPRERA (2013). http://www.euprera.org/?p=67. [09.07.2013].

IPR-INSTITUTE FOR PR; O'NEIL, J. (2012). "Resources for PR Educators for Teaching Research, Measurement and Evaluation". IPR Education in PR Comission. http://www.instituteforpr.org/iprwp/wpcontent/uploads/ONeil- resources-educators-FINAL-02-01-12.pdf. [26.06.2013].

KRIPPENDORFF, K. (1990). Metodología de análisis de contenido. Teoría y práctica. Barcelona: Paidós.

L'ETANG, J. (1999). "PR Education in Britain: An Historical Review in the Context of Professionalism". En: Public Relations Review, 25, pp. 261-289.

L'ETANG, J.; PIECKZA, M. (2006). "Public Relations Education". En L'ETANG, J.; PIECKZA, M. (eds.), Public Relations: Critical debates and contemporary practice. Hillsdale (NJ): Erlbaum, pp. 433-442.

LALUEZA, F. (2009). "Lo que hay que tener. Estudio comparativo de las competencias requeridas al profesional de las R.P. en el modelo formativo estadounidense y en el modelo formativo español post-Bolonia", Actas del IV Congreso Internacional de AIRP, Abril. Barcelona.

LEDINGHAM, J.A.; BRUNING, S.D. (2000). Public relations as relationship Management. A relational approach to the study and practice of Public Relations. Mahwah (NJ): Erlbaum.

LEEDS METROPOLITAN UNIVERSITY (2008). "Toward a Global Curriculum: A Summary of Concerning PR Education, Professionalism and Globalization". Global Alliance. http://www.globalalliancepr.org/website/sites/default/files/fedeles/Global\%20Curriculum/Towards\%20a\%20Global\%20Curriculum\%20-\%20 final\%20Feb1.pdf. [11.06.2013].

LEY ORGÁNICA 4/2007, de 12 de abril, por la que se modifica la Ley Orgánica 6/2001, de 21 de diciembre, de Universidades, $B O E \mathrm{n}^{\mathrm{o}} 89$ de 13.04.07, 16.242. http://www.aneca.es/var/media/158369/e02bleyorganica42007de12abrilmodificalou.pdf. [14.07.2013].

MAGALlÓN, S. (1998). Definición empírico-operativa del «practicum» de los estudiantes universitarios de R.P. y su vinculación con la realidad profesional en Cataluña, 1992-1997. Tesis doctoral. Bellaterra: Universidad Autónoma de Barcelona.http://www.tdx.cat/TDX-0622109-171218. [22.08.2013]. 
MATILLA, K.; HERNÁNDEZ, S. (2013). "Bolonia 3er. Año: los estudios universitarios de R.P. en Cataluña en el curso 2012-13". VIII Congreso AIRP, Abril. Zaragoza.

(2012). "Bolonia $2^{\circ}$ año: los estudios universitarios de R.P. en Cataluña en el curso 2010-2011". En: Revista Internacional de Relaciones Públicas, 4(2), pp. 247-276. http://dx.doi.org/ 10.5738/RIRP-4-2012-11-247-276. [29.06.2013].

(2011). "Bolonia 1r año: los estudios universitarios de R.P. en Cataluña en el curso 2010-2011”. En: Icono 14, Actas VI Congreso AIRP. Mayo. Vigo. http://es.scribd. $\mathrm{com} / \mathrm{doc} / 54467550 /$ Actas-6-VI-Congreso-Internacional-de-Investigacion-y-Relaciones-Publicas. [29.06.2013].

(2010a). "Las asignaturas de Relaciones Públicas en el marco del EEES". En SIERRA SÁNCHEZ, J. (coord..), Los estudios de Ciencias de la Comunicación en el EEES. Madrid: Fragua, pp. 293-307.

MATILLA, K.; XIFRA, J. (2009). "Comunicación Corporativa y R.P.: un proyecto de aproximación universidad/profesión en Cataluña, en el contexto de integración al Espacio Europeo de Enseñanza Superior”. En: Razón y Palabra, ${ }^{\circ} 70$, Noviembre-Febrero. http://www.razonypalabra.org.mx/Articulo\%201\%20RyPKMatillaJXifra110709.pdf. [29.06.2013].

MATILLA, K.; LOSADA, J.C.; HERNÁNDEZ, S. (2010b). "La oferta formativa de R.P. en los nuevos Grados universitarios en España". En: Pangea, 1. http:// revistapangea.org/2010/12/05/01-01-107/. [29.06.2013].

MINISTERIO DE EDUCACIÓN (2006). Propuestas para la renovación de las metodologías educativas de la universidad. Consejo de Coordinación Universitaria de la Secretaría de Estado de Universidades e Investigación. http://www.educacion.es/dctm/mepsyd/educacion/universidades/estadisticasinformes/estadisticas/ propuestarenovacion.pdf?documentId=0901e72b80048b70. [13.08.2013].

(2010). Datos y cifras del Sistema Universitario. Curso 2009-10. Secretaría General de Universidades, Subdirección General de Análisis, Estudios y Prospectiva Universitaria.http://www.educacion.es/dctm/ministerio/educacion/universidades/ estadisticas-informes/datos-cifras/2010-datos-y-cifras-09-10.pdf? documentI$\mathrm{d}=0901 \mathrm{e} 72 \mathrm{~b} 8009 \mathrm{f} 6 \mathrm{bb}$. [13.08.2013].

MOLLEDA, J.C. (2000). "International Paradigms: The Latin American School of PR”. En: Journalism Studies, 2, pp. 513-30.

PARDO, J.C.; GARCÍA TOBÍO, A. (2008). "El Espacio Europeo de Educación Superior: la razón instrumental de una universidad al servicio de la empresa". En: Innovación Educativa, 18, pp. 97-124.

PARÉS I MAICAS, M. (2006): "Las Relaciones Públicas, una ciencia social”. En: Anàlisi, 34.

PÉREZ GARCÍA, F.; SERRANO MARTÍNEZ, L. (dirs.) (2012). Universidad, universitarios y productividad en España. Bilbao: Fundación BBVA.http:// www. aqu.cat/doc/doc_92831049_1.pdf. [01.08.2013].

PIÑUEL RAIGADA, J.L.; LŌZANO A.; GARCÍA JIMÉNEZ, A. (eds.) (2011). "Investigar la Comunicación en España". Actas del 1r. Simposium de Grupos Consolidados de Investigación en Comunicación, Vol. I del 1r. Congreso Nacional 
de Metodología de la Investigación en Comunicación. Madrid: AE-IC-Asociación Española de Investigación en Comunicación. http://es.scribd.com/doc/127756071/ InvestigarlaComunicacionenEspana-Abril2011. [13.08.2013].

PIROZEK, P.; HESKOVA, M. (2003). "Approaches to and Instruments of PR: Higher Education in the Czech Republic". En: Higher Education in Europe, 28, pp.487-494. http://www.ingentaconnect.com/content/routledg/.../00000028/00000004. [09.07.2013].

SPACAL, T. (2007). "PR, Marketing, and Communications in Eastern European Universities". En JAMMERNEGG, I. (ed.), Setting Corporate Standards across Cultural Boundaries. Italy: Forum Editrice, pp. 42-54. http://www.forumeditrice. it. [23.07.2013].

SRIRAMESH, K. (2002). "The Dire Need for Multiculturalism in PR Education: An Asian Perspective”. En: Journal of Communication Management, 7, pp. 54-70.

THE MELBOURNE MANDATE (2012). Global Alliance. http://melbournemandate.wordpress.com/. [16.08.2013].

TILSON, D.J.; PÉREZ, P.S. (2003). "Public Relations and the New Golden Age of Spain: a Confluence of Democracy, Economic Development and the Media". En: Public Relations Review, 29(2), pp. 125-143.

TOTH, E.L.; ALDOORY, L. (2010). "A First Look: an In-Depth Analysis of Global PR Education. PR Curriculum and Instructor for 20 Countries". PRSA Foundation - Global Alliance for PR and Communications Management - Commission on PR Education, March. http://www. commpred.org/_uploads/ report4-full.pdf. [22.08.2013].

WRIGHT, D.K.; TURK, J.V. (2007). "Public Relations Knowledge and Professionalism: Challenges to Educators and Practitioners". En TOTH, E.L. (ed.), The Future of Excellence in Public Relations and Communication Management: Challenges for the Next Generation. Mahwah (NJ): Erlbaum, pp. 571-588.

XIFRA, J. (2007). "Undergraduate PR Education in Spain: Endangered Species?". En: Public Relations Review, 33, pp. 206-213.

ZHANG, A. (2009): Understanding Chinese PR Education: A Critical and Cultural Perspective. University of Maryland, College Park. http://hdl. handle. net/1903/10238. [06.06.2013].

ZLATEVA, M. (2003). "PR Education. An Instrument for the Transformation and Development of Human Resources". En: Higher Education in Europe, 28(4), December, pp. 511-518. http://www.ingentaconnect.com/content/routledg/.../00000028/00000004. [06.06.2013].

\section{Los autores}

Kathy Matilla. Doctora por la Universidad Ramon Llull (Barcelona), en cuya Facultad de Comunicación imparte docencia. Como consultora estratégica en R.R.P.P. ha prestado sus servicios a clientes como Akzo, Bridgestone, Bristol-Myers Squibb, 
Firestone, Gallina Blanca, Generalitat de Cataluña, Hoechst, La Caixa, Lancaster, Legrain, Louis Vuitton-Moët-Hennesy, Mahou-San Miguel, Nokia, Partido Demócrata (E.E.U.U.), Pfizer, Pharmacia \& Upjohn, Rolex, Revlon, Satélite ASTRA, o W.R. Grace. Preside FISEC-Foro Iberoamericano sobre Estrategias de Comunicación y es Vocal de las Juntas Directivas de Dircom Cataluña y de A.I.R.P.-Asociación de Investigadores en Relaciones Públicas. Anteriormente fue Vicedecana de R.R.P.P. del Colegio de Publicitarios y Relaciones Públicas de Cataluña.

Salvador Hernández Martínez. Doctor por la Universidad de Sevilla y acreditado por la ANECA como ayudante doctor y contratado doctor. Director de la Escuela Superior de Protocolo y Relaciones Institucionales de la Universidad Católica San Antonio (Murcia), donde ejerce como profesor de la Facultad de Comunicación desde 1998. Autor de una monografía, de numerosos capítulos de libros, artículos en revistas y ponencias en congresos nacionales e internacionales sobre Relaciones Públicas y Protocolo, es Secretario General de O.I.C.P.-Organización Internacional de Protocolo, miembro de la Junta Directiva de A.I.R.P.-Asociación de Investigadores en Relaciones Públicas y Vicepresidente de A.R.P.A.-Asociación de Relaciones Públicas y Protocolo de Andalucía. 